\title{
Rigidity constants from mean-field models
}

Cite as: J. Chem. Phys. 112, 2980 (2000); https://doi.org/10.1063/1.480872

Submitted: 24 August 1999 . Accepted: 12 November 1999 . Published Online: 31 January 2000

S. M. Oversteegen, and E. M. Blokhuis

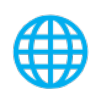

\section{ARTICLES YOU MAY BE INTERESTED IN}

Communication: Tolman length and rigidity constants of water and their role in nucleation The Journal of Chemical Physics 142, 171103 (2015); https://doi.org/10.1063/1.4919689

Tolman length and rigidity constants of the Lennard-Jones fluid

The Journal of Chemical Physics 142, 064706 (2015); https://doi.org/10.1063/1.4907588

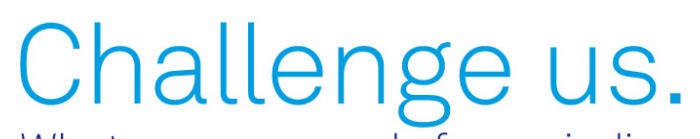

What are your needs for periodic signal detection?
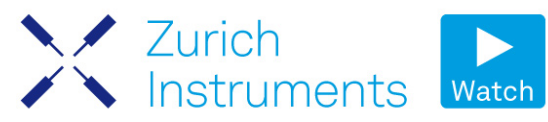

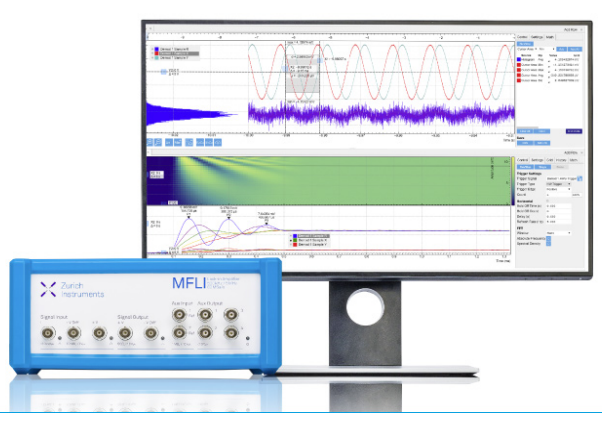

112,2980 


\title{
Rigidity constants from mean-field models
}

\author{
S. M. Oversteegen a) \\ Laboratory of Physical Chemistry and Colloid Science, Wageningen Agricultural University, \\ P.O. Box 8038, 6700 EK Wageningen, The Netherlands
}

E. M. Blokhuis

Colloid and Interface Science, Leiden Institute of Chemistry, Gorlaeus Laboratories, P.O. Box 9502, 2300 RA Leiden, The Netherlands

(Received 24 August 1999; accepted 12 November 1999)

\begin{abstract}
The interfacial tension of the planar interface and rigidity constants are determined for a simple liquid-vapor interface by means of a lattice-gas model. They are compared with results from the van der Waals model and from an analytical expansion around the critical point. The three approaches are in agreement in the regions where these theories apply. (C) 2000 American Institute of Physics. [S0021-9606(00)51106-4]
\end{abstract}

\section{INTRODUCTION}

The curvature of interfaces determines the physics of many systems to a large extent. This was already realized by Gibbs, but he gave some reasoning to ignore the curvature terms in the thermodynamic description. ${ }^{1}$ Five decades later, Tolman addressed this issue again and derived from the Gibbs adsorption equation a first-order curvature correction to the interfacial tension of a simple liquid-vapor interface, later known as the so-called Tolman length. ${ }^{2}$ From a mechanical point of view, Helfrich later introduced a more generally applicable correction to the free energy of an interface that was up to second order in the curvature. ${ }^{3}$ In terms of the interfacial tension $\gamma$, this description reads

$$
\gamma(J, K)=\gamma^{0}-k_{c} J_{0} J+\frac{1}{2} k_{c} J^{2}+\bar{k} K,
$$

where $J$ is the total curvature, $K$ the Gaussian curvature, and $\gamma^{0}$ the interfacial tension of the planar interface. The saddlesplay modulus $\bar{k}$ determines the topology of the interface rather than its rigidity, which is in turn determined by the bending modulus $k_{c}$. The bending modulus times the spontaneous curvature, $J_{0}$, is closely related to the Tolman length.

Many suggestions have been made to determine the aforementioned constants from a molecular model. ${ }^{4-7} \mathrm{Re}-$ cently, another suggestion has been made ${ }^{8}$ which combines the thermodynamic and mechanical route, as shown in Sec. II. In Sec. III we illustrate the derived equations by means of a mean-field lattice model for a simple liquid-vapor interface. These results are checked in Sec. IV by the well-known van der Waals theory, which has been employed before for simple interfaces. ${ }^{9}$ Finally, the results are discussed in Sec. V.

\footnotetext{
${ }^{a)}$ Present address: Van't Hoff Laboratory for Physical and Colloid Chemistry, Debye Research Institute, Utrecht University, P.O. Box 80051, 3508 TB Utrecht, The Netherlands. Electronic mail: m.oversteegen@chem.uu.nl
}

\section{THERMODYNAMICS OF CURVED INTERFACES}

In a phase-separated system, the interface between the phases is usually not infinitely sharp, owing to the thermal motion of the molecules. Following the Gibbs convention for a two-phase system, the system is split up in two bulk phases $\alpha$ and $\beta$ divided by an infinitely thin interface at an arbitrary position $R_{s}$. All bulk values are extrapolated up to the interface, and deviations from the bulk values, the excess amounts, are attributed to the interface. ${ }^{10}$ The curvature of the interface is determined by the total curvature $J=1 / R_{1}$ $+1 / R_{2}$ and the Gaussian curvature $K=1 / R_{1} R_{2}$, respectively, where $R_{1}$ and $R_{2}$ are the local radii of curvature of the interface at $R_{s}$. This introduces two new degrees of freedom so that the change of the grand potential of the interface $\Omega^{s}$ is given by

$$
d \Omega^{s}=-S^{S} d T-\mathbf{n}^{s} \cdot d \boldsymbol{\mu}+\gamma d A+A \mathrm{C}_{1} d J+A \mathrm{C}_{2} d K,
$$

where $S^{s}$ is the interfacial entropy, $T$ the absolute temperature, $\boldsymbol{\mu}$ the set of chemical potentials of all $\mathbf{n}^{s}$ molecules adsorbed at the interface of area $A$. The terms conjugated to the curvatures are the so-called bending stress $\mathrm{C}_{1}$ and torsion stress $C_{2}$, respectively. ${ }^{11}$ Integration of Eq. (2) and subsequent differentiation provides us the most complete version of the well-known Gibbs adsorption equation

$$
d \gamma=-\frac{S^{s}}{A} d T-\boldsymbol{\Gamma} \cdot d \boldsymbol{\mu}+\mathrm{C}_{1} d J+\mathrm{C}_{2} d K,
$$

where $\boldsymbol{\Gamma} \equiv \mathbf{n}^{s} / A$ is the adsorbed amount.

We next consider the work needed to bend a planar interface to a certain curvature $(J, K)$. This requires integration of the Gibbs adsorption equation, Eq. (3). At constant temperature and chemical potentials, this reads

$$
\int_{\gamma^{0}}^{\gamma(J, K)}\left(d \gamma^{\prime}\right)_{T, \mu}=\int_{0}^{J} C_{1} d J^{\prime}+\int_{0}^{K} C_{2} d K^{\prime} .
$$

When the chemical potentials are a function of the applied curvature, they are not an independent degree of freedom since their change is then already accounted for by the bending and torsion stress. For small deviations from the planar 
interface, the integrals on the right-hand side of Eq. (4) can be approximated by series expansion up to second order in the curvature, which yields

$$
\gamma(J, K) \approx \gamma^{0}+\mathrm{C}_{1}^{0} J+\frac{1}{2}\left(\frac{\partial \mathrm{C}_{1}}{\partial J}\right)^{0} J^{2}+\mathrm{C}_{2}^{0} K,
$$

where the superscript 0 denotes evaluation at the planar interface. This is very reminiscent of the expression Helfrich gave from mechanical arguments for a phenomenological description of the undulation of lipid bilayers. ${ }^{3}$ Comparison of Eq. (5) with the Helfrich equation, Eq. (1), yields for the rigidity constants

$$
\begin{aligned}
& -k_{c} J_{0}=\mathrm{C}_{1}^{0}=\left(\frac{\partial \gamma}{\partial J}\right)_{T, \boldsymbol{\mu}, K}^{0}, \\
& k_{c}=\left(\frac{\partial \mathrm{C}_{1}}{\partial J}\right)^{0}=\left(\frac{\partial^{2} \gamma}{\partial J^{2}}\right)_{T, \boldsymbol{\mu}, K}^{0}, \\
& \bar{k}=\mathrm{C}_{2}^{0}=\left(\frac{\partial \gamma}{\partial K}\right)_{T, \boldsymbol{\mu}, J}^{0},
\end{aligned}
$$

where we have used the total differential Eq. (3) for the definitions of the bending and torsion stress. We have linked the thermodynamics of curved interfaces to the rigidity constants. In order to derive these constants from a molecular model, we are interested in finding mechanical expressions for them. These are obtained in a quasithermodynamic way as proposed by Buff. ${ }^{12}$

From standard thermodynamics and Eq. (2), it is found that the total grand potential of the system reads

$$
\Omega=-p^{\alpha} V^{\alpha}-p^{\beta} V^{\beta}+\gamma A,
$$

where $p^{\alpha}$ and $p^{\beta}$ are the bulk pressures of the respective bulk phases of volume $V^{\alpha}$ and $V^{\beta}$, respectively. The actual pressure is obviously a continuous function through space, rather than a step function. However, due to the Gibbs convention, the bulk pressures $p^{\alpha}$ and $p^{\beta}$ have been extrapolated up to the interface. The total difference between the actual and the extrapolated pressure must be assigned to the interface. Since the only interfacial work is lateral, the excess of the tangential pressure profile $p_{T}(\mathbf{r})$ must constitute the interfacial mechanical work $^{12}$

$$
\gamma A=\int_{V^{\alpha}}\left(p^{\alpha}-p_{T}(\mathbf{r})\right) d \mathbf{r}+\int_{V^{\beta}}\left(p^{\beta}-p_{T}(\mathbf{r})\right) d \mathbf{r} .
$$

Using the principle of parallel interfaces, the volume element can be written as $d \mathbf{r}=A(r) d r$, where the area $A(r)$ at any position $r$ can be given analytically relative to the interfacial area $A$ at $R_{s}$ by $A(r)=A\left\{1+\left(r-R_{s}\right) J\right.$ $\left.+\left(r-R_{s}\right)^{2} K\right\} .{ }^{13,14}$ Substitution into Eq. (8) gives for the interfacial tension

$$
\gamma=\mathbb{P}_{0}+\mathbb{P}_{1} J+\mathbb{P}_{2} K,
$$

where we introduced the zeroth, first, and second bending moments

$$
\mathbb{P}_{0} \equiv \int\left(p^{\alpha \beta}-p_{T}(r)\right) d r,
$$

$$
\begin{aligned}
& \mathbb{P}_{1} \equiv \int\left(r-R_{s}\right)\left(p^{\alpha \beta}-p_{T}(r)\right) d r, \\
& \mathbb{P}_{2} \equiv \int\left(r-R_{s}\right)^{2}\left(p^{\alpha \beta}-p_{T}(r)\right) d r,
\end{aligned}
$$

where, in turn, the step function $p^{\alpha \beta} \equiv p^{\alpha} \theta\left(R_{s}-r\right)+p^{\beta} \theta(r$ $-R_{s}$ ) has been introduced, using the Heaviside step function $\theta\left(r-R_{s}\right)$.

Now that we have a mechanical expression for the interfacial tension, we find from Eqs. (6) and (9) the following mechanical expressions for the rigidity constants:

$$
\begin{aligned}
& -k_{c} J_{0}=\mathrm{P}_{1}^{0}+\left(\frac{\partial \mathrm{P}_{0}}{\partial J}\right)_{T, K}^{0}, \\
& k_{c}=2\left(\frac{\partial \mathbb{P}_{1}}{\partial J}\right)_{T, K}^{0}+\left(\frac{\partial^{2} \mathbb{P}_{0}}{\partial J^{2}}\right)_{T, K}^{0}, \\
& \bar{k}=\mathbb{P}_{2}^{0}+\left(\frac{\partial \mathrm{P}_{0}}{\partial K}\right)_{T, J}^{0} .
\end{aligned}
$$

In the next section, these rigidity constants are determined for a simple liquid-vapor interface by means of a lattice-gas model.

\section{LATTICE-GAS MODEL FOR CURVED INTERFACES}

In order to have an easily accessible partition function, space is divided into sites (cells) with equal volume $\mathrm{v}_{0}$ $=l^{3}$, where $l$ is a characteristic molecular size. From the lattice formed in this way, only $z=1, \ldots, M$ parallel layers are considered of $L(z)$ sites each. We can form planar, cylindrical, and spherical lattices this way. In the layers $z \leqslant 1$ and $z \geqslant M$ bulk conditions prevail. Imposing a mean-field approximation, it can be derived from standard statistical thermodynamics that for a one-component system the grand potential is given by ${ }^{15}$

$$
\frac{\Omega[\phi]}{k_{B} T}=\sum_{z=1}^{M} L(z)[f(\phi(z))-\mu \phi(z)],
$$

where $k_{B}$ is Boltzmann's constant, $\phi(z) \equiv N(z) / L(z)$ the density of the molecules in layer $z$ and the free energy density $f(\phi)$,

$$
\begin{aligned}
f(\phi)= & \phi \ln \phi+(1-\phi) \ln (1-\phi)-\phi \chi\langle\phi\rangle \\
& +\frac{1}{2} \chi\{\phi+\langle\phi\rangle\} .
\end{aligned}
$$

The interaction equals $\chi k_{B} T$ per $Z$ contacts, where $Z$ is the coordination number. The so-called contact fraction accounts for the mean-field interactions with adjacent lattice layers

$$
\langle\phi(z)\rangle \equiv \lambda_{-1}(z) \phi(z-1)+\lambda_{0}(z) \phi(z)+\lambda_{1}(z) \phi(z+1) .
$$

The transition probability $\lambda_{0}(z)$ is the fraction of adjacent sites $Z$ in layer $z$, whereas $\lambda_{-1}(z)$ and $\lambda_{1}(z)$ are the fractions of adjacent sites in the previous and next layer, respectively. Obviously, in a planar lattice $\lambda_{-1}=\lambda_{1}$. The sum of the transition probabilities equals unity, so in the bulk $[\phi(z-1)$ 


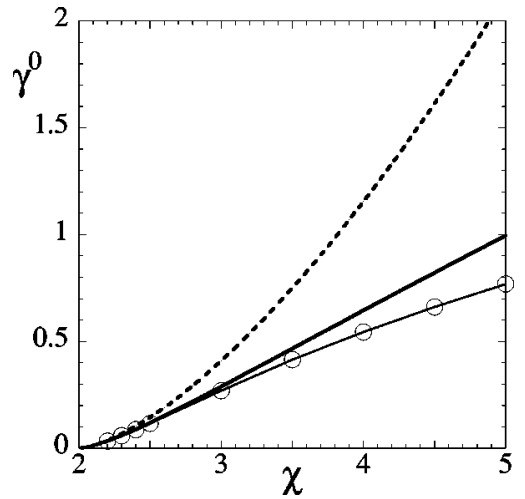

FIG. 1. The interfacial tension of the planar interface as function of the interaction parameter in scaled units such that $k_{B} T=1$ and $l=1$. The symbols are determined by the lattice-gas model using a simple cubic lattice. The solid line gives the corresponding van der Waals description [Eq. (22a)], whereas the dashed line gives the asymptotic values [Eq. (29a)].

$=\phi(z)=\phi(z+1)]$ the contact fraction reduces to the bulk density. Since $\Sigma_{z} L(z)=V / \mathrm{v}_{0}$, Eq. (13) reduces in the bulk to the Flory-Huggins free energy. ${ }^{6}$

The chemical potential in Eq. (12) can be regarded as a Lagrange multiplier that minimizes the free energy at the constraint of fixed number of molecules. From its thermodynamic definition, $\mu \equiv(\partial F / \partial N)_{T, V}$ and Eq. (13), the chemical potential reads

$$
\frac{\mu}{k_{B} T}=\ln \left(\frac{\phi(z)}{1-\phi(z)}\right)-2 \chi\langle\phi(z)\rangle+\chi .
$$

Although the individual terms of Eq. (15) are a function of $z$, the equilibrium chemical potential must be constant throughout the lattice. A density profile must be found that satisfies the criterion that the chemical potential in each layer equals the bulk chemical potentials. It is now easily seen that this density profile minimizes the free energy.

The grand potential density, the terms within square brackets of Eq. (12), is now identified as the tangential pressure profile. ${ }^{12,15}$ For both the bulk phases $\alpha$ and $\beta$, the bulk pressures are found from Eq. (12), Eq. (15), and $\Omega^{b}$ $=-p^{b} V^{b}$, where $b=\alpha$ or $\beta$

$$
\frac{p^{b} \mathrm{v}_{0}}{k_{B} T}=-\ln \left(1-\phi^{b}\right)-\chi \phi^{b 2}
$$

For $\chi>\chi_{c}$ this gives the familiar van der Waals loop. The spinodals given by $\left(\partial p^{b} / \partial\left(1 / \phi^{b}\right)\right)_{T}=0$ merge at the critical point, which yields from Eq. (16) that $\chi_{c}=2$ and $\phi_{c}^{\alpha}=\phi_{c}^{\beta}$ $=1 / 2$.

When $\phi^{\alpha}=\phi(1)$ and $\phi^{\beta}=\phi(M)$ are applied, the excess pressure profile follows directly from Eqs. (12) and (16). Consequently, the respective bending moments can be determined for the lattice-gas model, as outlined in Appendix A.

The interfacial tension of the planar interface $\gamma^{0}$ can be straightforwardly identified with the zeroth bending moment, ${ }^{16}$ as it also follows directly from Eq. (9). This has been done for several values of the interaction parameter $\chi$ $>\chi_{c}$, as shown by the symbols in Fig. 1 for a simple cubic lattice, i.e. $\lambda_{-1}=\lambda_{1}=\frac{1}{6}$ in units such that $k_{B} T=1$ and $l=1$. Since the interfacial width diverges at the critical tempera- ture, the interface and the interfacial tension vanish. Far away from $\chi_{c}$ the interface becomes sharper such that the interfacial entropy becomes less important and eventually the interfacial tension is completely energetic: $\gamma^{0}=\chi \lambda_{1}$. Note that the interfacial tension of the planar interface is independent of the choice of the position of the dividing plane since $p^{\alpha}=p^{\beta}$. This choice is, however, important for curved interfaces. Henceforth, we will take the Gibbs-dividing or equimolar plane to be the interface. This is the position where the excess number of molecules at the interface, i.e., the adsorbed amount, vanishes, i.e., $\Gamma=0$. This position is not necessarily an integer but can be in between two layer numbers.

In order to obtain the rigidity constants, as given by Eq. (11), knowledge of the curvature dependence of the bending moments is required. To that end, a certain pressure difference $p^{\alpha}-p^{\beta}=\gamma^{0} J$ is imposed, where $J$ is approximately the desired total curvature. The corresponding bulk chemical potential is found and consequently molecules are 'titrated' on the simple-cubic curved lattice until the chemical potential of the resulting phase separated system, as given by Eq. (15), equals the desired bulk chemical potential. Subsequently, the exact curvature $J$ is determined from the equimolar plane. The corresponding bending moments as given in Appendix A can now be calculated.

The spontaneous curvature $k_{c} J_{0}$ and the bending modulus $k_{c}$ can be determined strictly from the cylindrical lattice because in order to evaluate derivatives, as given in Eq. (11), the total curvature $J$ must be varied at constant Gaussian curvature $K$. The bending moments were determined for a cylindrical interface as a function of the total curvature as described above. A third-order polynomial was fit through the bending moments in order to evaluate the derivatives numerically. This way, we found from Eq. (11a) that the Tolman length or spontaneous curvature, $J_{0}$, vanishes for each value of the interaction parameter $\chi$. This should be the case from symmetry considerations ${ }^{17}$ since exchange of free volume and the monomeric species gives the same minimal free energy of the planar interface. This can easily be seen from Eq. (13) when species $[\phi(z)]$ are replaced by free volume $[1-\phi(z)]$.

The bending modulus $k_{c}$ was determined analogously as a function of the interaction parameter, as shown by the symbols in Fig. 2(a). The bending modulus of the lattice-gas model as given by Eq. (11b) vanishes in the critical point. For larger values of the interaction parameter, an interface is formed and the system is affected by the applied curvature. The bending modulus from the lattice-gas model goes through a minimum and for very large $\chi$ it appears to go to zero. This physically means that in the lattice-gas model the free energy of the interface for large $\chi$ is apparently dominated by the interfacial tension rather than curvature energy.

The saddle-splay modulus cannot be determined from a consideration of the cylindrical interface alone, since, according to Eq. (11c), $K$ must be varied at constant $J$. Neither can this be done from a spherical interface since $1 / R_{1}$ $=1 / R_{2}=1 / R_{s}$ such that $J$ and $K$ are no longer independent state variables: $K=\frac{1}{4} J^{2}$. Consequently, for a spherical interface one of the curvature terms in the starting thermody- 


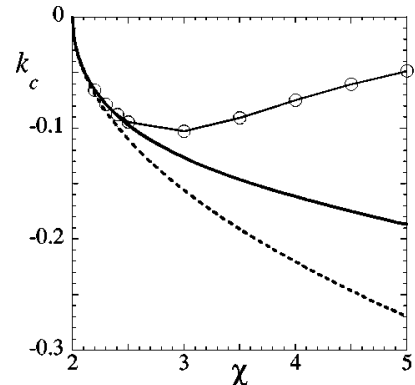

(a)

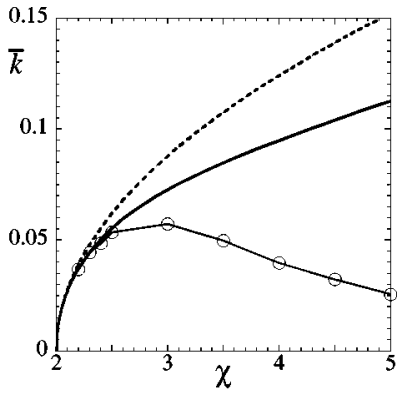

(b)
FIG. 2. (a) The bending modulus and (b) the saddle-splay modulus as function of the interaction parameter in scaled units such that $k_{B} T=1$ and $l$ $=1$. The symbols are determined by the lattice-gas model using a simple cubic lattice. The solid line gives the corresponding van der Waals description [Eqs. (22b) and (22d)], whereas the dashed line gives the asymptotic values [Eqs. (29b) and (29c)].

namic equation of the interface, Eq. (2), is redundant and the thermodynamic analysis should be gone through again. However, it is easily seen that this gives only one new state variable conjugate to the total curvature that incorporates both the bending and torsion stress. For the Helfrich equation we then also find only one "effective" modulus, $k_{c}+\frac{1}{2} \bar{k}$, given by Eq. (11b) where the respective bending moments are found from a spherical interface. From the effective bending modulus, determined completely analogously to the cylindrical geometry, the saddle-splay modulus $\bar{k}$ can be extracted since $k_{c}$ was already known from the cylindrical interface. The bending modulus, $k_{c}$, and the effective modulus, $k_{c}+\frac{1}{2} \bar{k}$, were determined from a third-order polynomial fit through the respective bending moments. Consequently, the extracted value for the saddle-splay modulus is subject to relatively much numerical noise. However, within the numerical accuracy, the same values could be found from a direct parabolic fit of Eq. (1) to the interfacial tension determined with Eq. (9). ${ }^{8}$ The results for the saddle-splay modulus are shown in Fig. 2(b) and give the same qualitative behavior as the bending modulus, albeit with a different sign and magnitude.

\section{VAN DER WAALS THEORY OF CURVED INTERFACES}

As outlined in Appendix B, the grand potential of a lattice-gas model, Eq. (12), can be regarded as the discretized version of the well-known free-energy functional as given by van der Waals. In units such that $k_{B} T=1, l=1$, and $\lambda_{1}$ $=1 / 6$, the continuous version of the grand potential, Eq. (12), then reads

$$
\Omega[\rho]=\int d \mathbf{r}\left[\frac{\chi}{6}|\nabla \rho(\mathbf{r})|^{2}+f(\rho)-\Delta \mu \rho(\mathbf{r})\right],
$$

with the free-energy density $f(\rho)$

$$
f(\rho)=\rho \ln (\rho)+(1-\rho) \ln (1-\rho)+\rho \chi(1-\rho)-\mu_{\mathrm{coex}} \rho,
$$

where $\rho(\mathbf{r})$ is the continuous density profile as opposed to the discrete density profile $\phi(z)$. The chemical potential $\Delta \mu \equiv \mu-\mu_{\text {coex }}$ is defined as the chemical potential distance to liquid-vapor coexistence and is used in the calculation to vary the curvature of the liquid-vapor interface.

As shown in Sec. II, the volume element $d \mathbf{r}$ depends on the geometry of the system. The Euler-Lagrange equation that minimizes the above grand potential in spherical geometry is given by

$$
\frac{\chi}{3} \rho_{s}^{\prime \prime}(r)=-\frac{4 \chi}{3} \frac{1}{r} \rho_{s}^{\prime}(r)+f^{\prime}\left(\rho_{s}\right)-\Delta \mu_{s},
$$

where $r$ is the radial distance. The subscript $s$ denotes the fact that we are considering a spherical interface, whereas the prime denotes the derivative with respect to its argument. In order to relate the grand potential to the curvature coefficients $\gamma^{0}, k_{c}$, and $\bar{k}$, an expansion is made in the reciprocal radius, $1 / R_{s}$, of the spherical droplet. The density and chemical potential expanded to first order are

$$
\begin{aligned}
& \rho_{s}(r)=\rho_{0}(r)+\rho_{1}(r) \frac{1}{R_{s}}+\mathcal{O}\left(\frac{1}{R_{s}^{2}}\right), \\
& \Delta \mu_{s}=\Delta \mu_{1} \frac{1}{R_{s}}+\mathcal{O}\left(\frac{1}{R_{s}^{2}}\right),
\end{aligned}
$$

where it can be shown that $\Delta \mu_{1}=2 \gamma^{0} / \Delta \rho,{ }^{17}$ with $\Delta \rho \equiv \rho_{l}$ $-\rho_{\mathrm{V}}$ the density difference between the liquid $\left(\rho_{l} \cong \phi^{\alpha}\right)$ and vapor $\left(\rho_{\mathrm{v}} \cong \phi^{\beta}\right)$ phase at coexistence. The Euler-Lagrange equation in Eq. (19) is also expanded to first order in the reciprocal radius

$$
\begin{aligned}
& \frac{\chi}{3} \rho_{0}^{\prime \prime}(z)=f^{\prime}\left(\rho_{0}\right), \\
& \frac{\chi}{3} \rho_{1}^{\prime \prime}(z)=-\frac{2}{3} \chi \rho_{0}^{\prime}(z)+f^{\prime \prime}\left(\rho_{0}\right) \rho_{1}(z)-\Delta \mu_{1},
\end{aligned}
$$

where we have defined $z \equiv r-R_{s}$, which must not be confused with the lattice index of Sec. III. Using the above differential equations, the grand potential of the interface can be extracted from Eq. (17) up to second order in the curvature. Comparison with the Helfrich equation, Eq. (1), yields the interfacial tension of the planar interface and rigidity constants expressed in terms of the density profiles $\rho_{0}(z)$ and $\rho_{1}(z)$ (Ref. 9)

$$
\begin{aligned}
& \gamma^{0}=\frac{\chi}{3} \int_{-\infty}^{\infty} d z\left[\rho_{0}^{\prime}\right]^{2}, \\
& k_{c} J_{0}=\frac{\chi}{3} \int_{-\infty}^{\infty} d z z\left[\rho_{0}^{\prime}\right]^{2}, \\
& k_{c}=-\frac{\chi}{6} \int_{-\infty}^{\infty} d z \rho_{1} \rho_{0}^{\prime}+\frac{\Delta \mu_{1}}{4} \int_{-\infty}^{\infty} d z z^{2} \rho_{0}^{\prime},
\end{aligned}
$$

$$
\bar{k}=\frac{\chi}{3} \int_{-\infty}^{\infty} d z z^{2}\left[\rho_{0}^{\prime}\right]^{2} .
$$

Similar expressions were previously derived from Landau theory. ${ }^{7,17}$ As also found in Sec. III, $k_{c}$ and $\bar{k}$, unlike $\gamma^{0}$ and $k_{c} J_{0}$, depend on the choice of the position of the dividing 
plane. In order to make a fair comparison with the lattice-gas model, the above expressions were derived by locating the interface at the equimolar plane, defined by

$$
\Gamma=\int d \mathbf{r}\left[\rho_{s}(z)-\rho_{\text {bulk }}(z)\right]=0,
$$

where $\rho_{\text {bulk }} \equiv \rho_{l} \theta(-z)+\rho_{\mathrm{v}} \theta(z)$. Expanded to first order in $1 / R_{s}$, Eq. (23) gives the following set of conditions for the profiles $\rho_{0}(z)$ and $\rho_{1}(z)$ :

$$
\begin{aligned}
& \int_{-\infty}^{\infty} d z\left[\rho_{0}(z)-\rho_{0, \mathrm{bulk}}(z)\right]=0, \\
& \int_{-\infty}^{\infty} d z\left[\rho_{1}(z)-\rho_{1, \mathrm{bulk}}(z)\right]=\int_{-\infty}^{\infty} d z z^{2} \rho_{0}^{\prime}(z) .
\end{aligned}
$$

With these two conditions, the differential equations in Eq. (21) have been solved numerically for the density profiles $\rho_{0}(z)$ and $\rho_{1}(z)$, using the explicit expression for $f(\rho)$ in Eq. (18). The resulting density profiles have then been inserted into the expression for the interfacial tension and rigidity constants as given by Eq. (22). The results of this numerical approach are shown as the solid lines in Figs. 1 and 2 .

Both the lattice-gas model and the van der Waals theory required a numerical solution of the density profiles. However, in the vicinity of the critical point, $\chi_{c}=2$, analytical solutions for the interfacial tension of the planar interface and rigidity constants can be derived. ${ }^{18}$ To that end, the density is expanded around the critical density, $\rho_{c}=\frac{1}{2}$. In particular, we can expand $f(\rho)$ to fourth order in $\left(\rho-\rho_{c}\right)$

$$
\begin{aligned}
f(\rho)= & f\left(\rho_{c}\right)-\left(\chi-\chi_{c}\right)\left(\rho-\rho_{c}\right)^{2}+\frac{4}{3}\left(\rho-\rho_{c}\right)^{4} \\
& +\mathcal{O}\left(\left(\rho-\rho_{c}\right)^{6}\right) .
\end{aligned}
$$

This is the familiar $\rho^{4}$-shape of the free-energy density used in the van der Waals theory for inhomogeneous systems. Solving the Euler-Lagrange equation for $\rho_{0}(z)$ in Eq. (21) with the above form for $f(\rho)$ yields the well-known hyperbolic-tangent profile ${ }^{6,19}$

$$
\rho_{0}(z)=\rho_{c}-\frac{\Delta \rho}{2} \tanh (z / 2 \xi),
$$

where the density difference $\Delta \rho$ and bulk correlation length $\xi$, which is a measure of the thickness of the interface, are given by

$$
\begin{aligned}
& (\Delta \rho)^{2}=\frac{3}{2}\left(\chi-\chi_{c}\right), \\
& \xi=\frac{1}{6} \sqrt{6}\left(\chi-\chi_{c}\right)^{-1 / 2} .
\end{aligned}
$$

Within the van der Waals theory, the values for the interfacial tension of the planar interface and rigidity constants have already been calculated, ${ }^{9}$ and we can simply insert the above expression for $\rho_{0}$ in Eq. (22). Using Eq. (28) for $\Delta \rho$ and $\xi$, this gives

$$
\gamma^{0}=\frac{\chi_{c}}{18} \frac{(\Delta \rho)^{2}}{\xi}=\frac{1}{6} \sqrt{6}\left(\chi-\chi_{c}\right)^{3 / 2},
$$

$$
\begin{aligned}
& k_{c}=-\frac{\chi_{c}}{54}\left(\pi^{2}-3\right)(\Delta \rho)^{2} \xi=\frac{-1}{108} \sqrt{6}\left(\pi^{2}-3\right)\left(\chi-\chi_{c}\right)^{1 / 2}, \\
& \bar{k}=\frac{\chi_{c}}{54}\left(\pi^{2}-6\right)(\Delta \rho)^{2} \xi=\frac{1}{108} \sqrt{6}\left(\pi^{2}-6\right)\left(\chi-\chi_{c}\right)^{1 / 2},
\end{aligned}
$$

where Eq. (29a) recovers the familiar mean-field result for the interfacial tension of the planar interface. ${ }^{19}$ The asymptotic expressions Eq. (29) are the dashed curves in Figs. 1 and 2.

\section{DISCUSSION}

From a quasithermodynamic route we derived mechanical expressions for the interfacial tension and the rigidity constants. These are evaluated from a lattice-gas model for a simple liquid-vapor interface. The results are given by the symbols in Figs. 1 and 2.

It is shown that the free energy of the one-component lattice-gas model is the discretized version of the wellknown van der Waals free-energy functional. The continuous version of this free energy is expanded up to second order in the curvature. Comparison with the Helfrich equation yields independent expressions for the interfacial tension of the planar interface and the rigidity constants in terms of (derivatives of) the density profile of the planar interface. These results are given by the solid lines in Figs. 1 and 2. Inserting a series expansion of the free-energy density up to fourth order around the critical density in the van der Waals expressions gives analytical expressions for the interfacial tension of the planar interface and the rigidity constants. These are given by the dashed lines in Figs. 1 and 2 .

The interfacial tension of the planar liquid-vapor interface, $\gamma^{0}$, has been studied extensively before. ${ }^{19}$ As clearly shown in Fig. 1, all three models have the same known mean-field behavior in the vicinity of the critical point $\chi_{c}$ $=2$. Away from the critical point, nonlocal effects must be included and the analytical solution, which does not account for that, deviates from the other two. Further away from the critical point $\left(\geq 1.2 \chi_{c}\right)$ the density profile in the interfacial region becomes steeper and the square gradient term in the van der Waals expression for the free energy is not sufficient to account for this rapidly varying density profile, higher order derivatives of the density profile should be included. This is accounted for in the lattice-gas expression for the free energy by the contact fraction, as can be seen from the truncated series expansion in Appendix B. Consequently, only the lattice-gas model gives the appropriate linear behavior far away from the critical point. This accounts for the differences between square gradient and the more exact integralfunctional theory as already known in the literature. ${ }^{20}$ Some progress can be made in the van der Waals description by adding a square Laplacian. ${ }^{9,21}$

We found that using Eq. (11a) in the lattice-gas model gave a vanishing Tolman length or spontaneous curvature, $J_{0}$. This must be the case from symmetry arguments ${ }^{17}$ and has also been found for the van der Waals theory, as follows from Eq. (22b). ${ }^{9}$ 
The bending modulus, $k_{c}$, determined from the latticegas model found from Eq. (11b), is in good quantitative agreement with the ones found from the van der Waals theory, Eq. (22c), up to the interaction parameter where the higher order derivatives of the density profile become impor$\operatorname{tant}\left(\chi \lesssim 1.2 \chi_{c}\right)$. The saddle-splay modulus, $\bar{k}$, found from Eq. (11c) is also in good qualitative agreement with the ones found from the van der Waals theory, Eq. (22d), in the region where the latter is valid.

In the region where all the above-mentioned theories are valid, they gave identical and physically relevant results for the interfacial tension of the planar interface and the bending constants. The van der Waals expressions, Eq. (22), are within a mean-field approximation for the pair density, consistent with the rigidity constants found from the virial route to the rigidity constants. ${ }^{9}$ Gompper et $a .^{7}{ }^{7}$ derived expressions that were very reminiscent of Eq. (22). They defined a free-energy density, in this particular case for instance $(\chi / 3)\left[\rho_{0}^{\prime}\right]^{2}$, as the tangential pressure profile, $p_{T}(z)$. Szleifer et al. did the same with their free-energy density ${ }^{5}$ to arrive at the same expressions for the rigidity constants as from the principle of virtual work. ${ }^{6}$ It is therefore concluded that the rigidity constants as given by Eq. (11) are consistent with all the previously mentioned models within the mean-field approximation.

\section{ACKNOWLEDGMENTS}

F.A.M. Leermakers is gratefully acknowledged for his discussions on the meaning of the bending modulus in simple liquid-vapor interfaces. The work of S.M.O. was supported by the Netherlands Organization for Scientific Research Chemical Sciences (NWO/CW). The research of E.M.B. has been made possible by a fellowship of the Royal Netherlands Academy of Arts and Sciences.

\section{APPENDIX A: DISCRETIZED BENDING MOMENTS}

In Sec. II the bending moments were found as given by Eqs. (10a), (10b), and (10c). Because the tangential pressure $p_{T}(r)$ was identified with the grand potential density from Eq. (12), it is constant within one layer. Consequently, a bending moment can be written as a sum over all layers. The zeroth bending moment is simply given by

$$
\begin{aligned}
\mathbb{P}_{0} & =\int\left(p^{\alpha \beta}-p_{T}(R)\right) d R \\
& =\sum_{z} \int_{z-1}^{z}\left(p^{\alpha \beta}-p_{T}(z)\right) d R=\sum_{z}\left(p^{\alpha \beta}-p_{T}(z)\right) .
\end{aligned}
$$

As before, $p^{\alpha \beta}$ equals $p^{\alpha}$ up to the dividing plane and $p^{\beta}$ beyond, where both bulk pressures are given by Eq. (16). The integral in Eq. (10a) is thus effectively replaced by a summation. However, this cannot be done for the first bending moment

$$
\begin{aligned}
\mathbb{P}_{1} & =\int\left(R-R_{s}\right)\left(p^{\alpha \beta}-p_{T}(R)\right) d R \\
& =\sum_{z} \int_{z-1}^{z}\left(R-R_{s}\right)\left(p^{\alpha \beta}-p_{T}(z)\right) d R \\
& =\sum_{z} \frac{1}{2} R^{2}-\left.R_{s} R\right|_{z-1} ^{z}\left(p^{\alpha \beta}-p_{T}(z)\right) \\
& =\sum_{z}\left(z-R_{s}-\frac{1}{2}\right)\left(p^{\alpha \beta}-p_{T}(z)\right) .
\end{aligned}
$$

Owing to the discretization, an extra factor $1 / 2$ comes in when replacing the integral by a summation. Analogously, it is found that the second bending moment in the lattice-gas model is given by

$$
\mathbb{P}_{2}=\sum_{z}\left(\left(z-R_{s}\right)^{2}-\left(z-R_{s}-\frac{1}{3}\right)\right)\left(p^{\alpha \beta}-p_{T}(z)\right) .
$$

\section{APPENDIX B: CONTINUOUS VERSION OF THE LATTICE GRAND POTENTIAL}

Since $L(z) \mathrm{v}_{0}$ is the volume of a layer, the summation over all layers of the grand potential density, as given by Eq. (12), is equivalent to a volume integral in continuous space. In that continuous limit, the discrete density profile $\phi(z)$ reduces to $\rho(\mathbf{r})$. For slowly varying densities, the continuous density profile may be approximated by a second-order series expansion around the local discrete densities. Substitution in the contact fraction, as defined in Eq. (14), gives

$$
\begin{aligned}
\langle\phi(z)\rangle \equiv & \lambda_{-1}(z) \phi(z-1)+\lambda_{0}(z) \phi(z)+\lambda_{1}(z) \phi(z+1) \\
\approx & \lambda_{-1}(z)\left(\rho(\mathbf{r})-l \nabla \rho(\mathbf{r})+\frac{1}{2} l^{2} \nabla^{2} \rho(\mathbf{r})\right) \\
& +\lambda_{0}(z) \rho(\mathbf{r}) \\
& +\lambda_{1}(z)\left(\rho(\mathbf{r})+l \nabla \rho(\mathbf{r})+\frac{1}{2} l^{2} \nabla^{2} \rho(r)\right) \\
= & \rho(r)+\lambda_{1} l^{2} \nabla^{2} \rho(r),
\end{aligned}
$$

where we implicitly assumed a planar lattice, i.e., $\lambda_{-1}$ $=\lambda_{1}$, and used the fact that the sum of the transition probabilities $\lambda$ equals unity. Integration by parts gives

$$
\begin{aligned}
& \sum_{z=1}^{M} L(z) \phi(z) \chi\langle\phi(z)\rangle \\
& \quad \cong \int_{\alpha}^{\beta} \frac{d \mathbf{r}}{\mathbf{v}_{0}}\left[\chi \rho(\mathbf{r})^{2}-\chi \lambda_{1} l^{2}|\nabla \rho(\mathbf{r})|^{2}\right],
\end{aligned}
$$

where use has been made of the fact that the density gradient vanishes in both bulk phases $\alpha(z \leqslant 1)$ and $\beta(z \geqslant M)$. Inserting this into Eq. (12) yields the well-known van der Waals free-energy functional for inhomogeneous systems. In this paper, we reduce this to the standard van der Waals form for the grand potential

$$
\begin{aligned}
\frac{\Omega}{k_{B} T}= & \int \frac{d \mathbf{r}}{\mathrm{V}_{0}}\left[\chi \lambda_{1} l^{2}|\nabla \rho(\mathbf{r})|^{2}+\rho(\mathbf{r}) \ln \rho(\mathbf{r})\right. \\
& \left.+(1-\rho(\mathbf{r})) \ln (1-\rho(\mathbf{r}))-\chi \rho(\mathbf{r})^{2}+\chi \rho(\mathbf{r})-\mu \rho(\mathbf{r})\right] .
\end{aligned}
$$


${ }^{1}$ J. W. Gibbs, The Scientific Papers (OxBow, Woodbridge, NJ, 1993), Vol. 1 , pp. 219-229.

${ }^{2}$ R. C. Tolman, J. Chem. Phys. 17, 333 (1949).

${ }^{3}$ W. Helfrich, Z. Naturforsch. C 28c, 693 (1973).

${ }^{4}$ E. M. Blokhuis and D. Bedeaux, Physica A 184, 42 (1992); Heterog. Chem. Rev. 1, 55 (1994).

${ }^{5}$ I. Szleifer, D. Kramer, A. Ben-Shaul, W. M. Gelbart, and S. A. Safran, J. Chem. Phys. 92, 6800 (1990).

${ }^{6}$ S. A. Safran, in Frontiers in Physics, edited by D. Pines (Addison-Wesley, Reading, MA, 1994), Vol. 90

${ }^{7}$ G. Gompper and S. Zschocke, Phys. Rev. A 46, 4836 (1992).

${ }^{8}$ S. M. Oversteegen, "Thermodynamic and Mechanical Properties of Curved Interfaces," Ph.D. thesis, Wageningen University, 1999.

${ }^{9}$ E. M. Blokhuis and D. Bedeaux, Mol. Phys. 80, 705 (1993).

${ }^{10}$ C. E. Reid, Chemical Thermodynamics (McGraw-Hill, Singapore, 1990).

${ }^{11}$ C. L. Murphy, "Thermodynamics of Low Tensions and Highly Curved Interfaces," Ph.D. thesis, University of Minnesota, 1966.
${ }^{12}$ F. P. Buff, J. Chem. Phys. 23, 419 (1955).

${ }^{13}$ J. Gaydos, L. Boruvka, Y. Rotenberg, P. Chen, and A. W. Neumann, in Applied Surface Thermodynamics, edited by A. W. Neumann and J. K. Spelt (Marcel Dekker, New York, 1996).

${ }^{14}$ V. S. Markin, M. M. Kozlov, and S. L. Leikin, J. Chem. Soc., Faraday Trans. 2 84, 1149 (1988).

${ }^{15}$ S. M. Oversteegen, P. A. Barneveld, F. A. M. Leermakers, and J. Lyklema, Langmuir 15, 8609 (1999).

${ }^{16}$ J. G Kirkwood and F. P. Bluff, J. Chem. Phys. 17, 338 (1949).

${ }^{17}$ M. P. A. Fisher and M. Wortis, Phys. Rev. B 29, 6252 (1984).

${ }^{18}$ A. E. van Giessen, E. M. Blokhuis, and D. J. Bukman, J. Chem. Phys. 108, 1148 (1998).

${ }^{19}$ J. S. Rowlinson and B. Widom, Molecular Theory of Capillarity (Clarendon, Oxford, 1982).

${ }^{20}$ B. Widom, J. Phys. Chem. 88, 6508 (1984).

${ }^{21}$ V. Romero-Rochín, C. Varea, and A. Robledo, Phys. Rev. A 44, 8417 (1991) 